\title{
FIRST RESULTS IN AUTOMATED PIPE EXCAVATION
}

\author{
by \\ William "Red" Whittaker, George Turkiyyah, François Bitz \\ John Balash, Rob Guzikowski, Bob Montgomery \\ Rasim Akdogan, Scott Swetz
}

\section{CIVIL ENGINEERING AND CONSTRUCTION ROBOTICS LABORATORY CARNEGIE-MELLON UNIVERSITY}

\author{
June 1985
}

\begin{abstract}
We have demonstrated in our laboratory the rudimentary sensor-driven excavation of pipes buried at shallow depths. Our system integrates sonar range sensing, a surface depth map, segmentation for pipe searching, simple digging strategies, distributed computing and off-line simulation. Our hardware is a manipulator mounted to a backhoe and a vacuum tool simulates the air jet tool intended for field excavation.
\end{abstract}




\section{PIPE EXCAVATION IN CONTEXT}

We are researching the Robotic EXcavation (REX) of buried utility piping without the intervention of an operator in the control loop. One motivation for unmanned excavation is the hazard posed to humans from explosions that occcur during the manual excavation of gas utilities. An ultimate motivation is improved efficiency, decreased cost and increased productivity for s.nall scale digging. REX aspires to integrate the following elements to excavate pipes :

- Subsurface pre-mapping of pipes, structures and other objects using available utility records and ground-penetrating sensors. Magnetic sensing is the leading candidate for pre-mapping metallic pipes.

- Primary excavation for gross access near to the target pipes. Trenching and auguring are the leading candidates for this operation. Besides coarse excavation, the primary excavator is responsible for clearing material that is dislodged during secondary excavation. Vacuum transport is a candidate for material removal.

- Secondary excavation is the fine and benign digging that progresses from the primary excavation to clear piping. Secondary excavation is guided from a sensor-driven world map. A supersonic air jet is the tooling of current choice.

The work that follows is specific to the REX secondary excavation, but is easily extensible to sensor-driven sandblasting, material spraying or jet cleaning.

\section{OVERVIEW}

This paper describes the first complete system as an evolution towards autonomous excavation of utility pipelines : it covers all the levels of the task execution from high level planning to sensing, trajectory generation, and the low level control of joint actuators: The approach to the problem is breadth first : A complete working system is implemented while refinements and optimizations are deferred for follow-on research.

We have demonstrated the rudimentary excavation of pipes buried at shallow depths in our laboratory testbed. Our actuation hardware (See figure2) consists of a manipulator mounted on a backhoe. A vacuum tool (simulating the air jet cutter intended by REX for actual excavation) removes vermiculite (simulating the soil encountered in actual excavation). A single sonar sensor mounted on the tool gathers range data to 
build the surface models that drive the system.

The system (See figure 1) initializes by scanning the domain to build a model of surface topography. This model is searched for the presence of target objects and obstacles. The surface is traversed by the airjet which erodes away the surface in layers. Pipes that appear in the course of excavation trigger pipe-specific digging strategies. All digging actions for a layer are generated by operations on the surface model, which is updated after erosion of each layer. All motions are concurrently monitored in animated simulation on a graphics workstation.

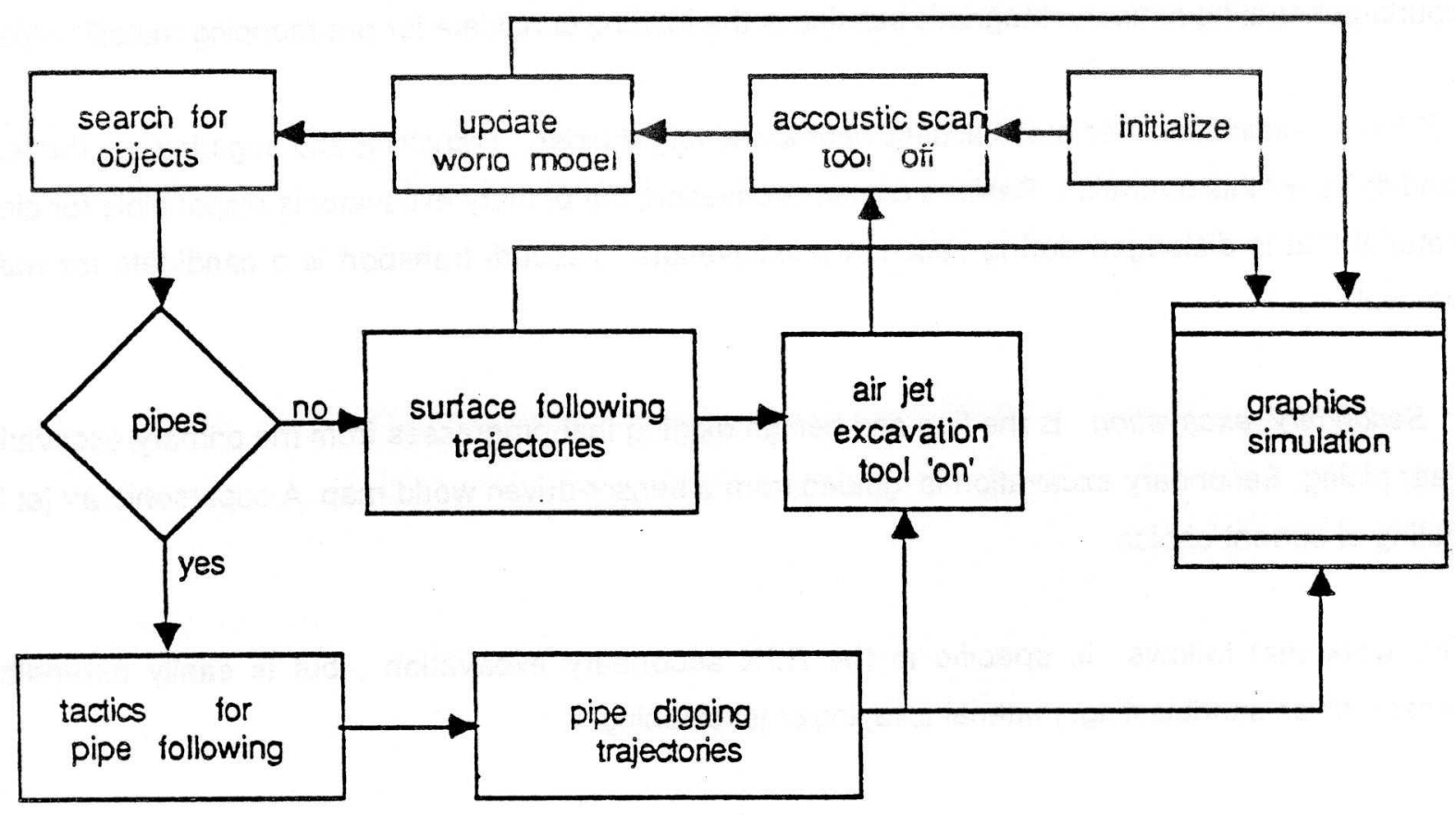

Fig 1. System block diagram

The remainder of this paper describes the hardware and software elements of secondary excavation and the demonstration that is our proof-of-concept. 


\section{SECONDARY EXCAVATION HARDWARE}

The secondary excavation hardware (fig 2) consists of a servo controlled backhoe for gross positioning, a backhoe-mounted manipulator for deploying a digging tool and a vacuum nozzle to simulate tool action. The system excavates a testbed that simulates an excavation site in the lab. A single board computer interfaces these devices and controls the excavation process.

A conventional backhoe, modified with servo valves and joint resolvers, provides base support for the manipulator which is mounted in place of the backhoe bucket. The swing joint of the backhoe is currently 'fixed to reduce positioning backlash. The gross motions of the backhoe position the arm in local regions or "patches" where scanning and digging operations require only manipulator articulations. Excavation is performed sequentially on patches within the site.

The manipulator is an elbow manipulator of the type used for subsea teleoperation. It was modified for increased envelope and uncluttered profile. It exhibits a payload of $300 \mathrm{lbs}$ at full extension and over 1000 lbs in its optimal lifting configuration. A master arm is provided as a human interface for manipulator setup and for error recovery. Together the backoe and 6-degree of freedom manipulator provide 9-degrees of freedom for tool positionning and orientation.

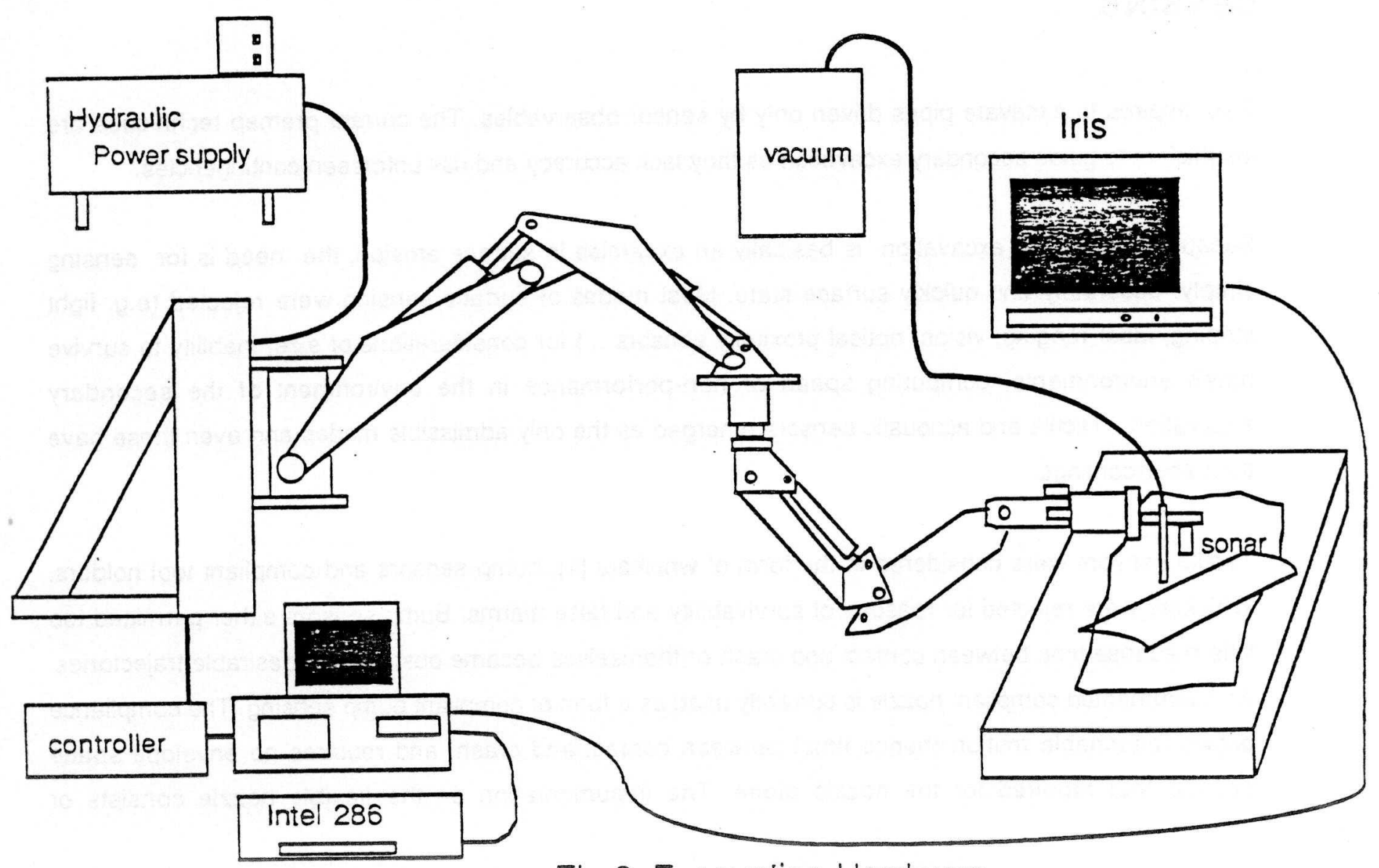

Fig 2 Excavation Hardware 
Our laboratory excavation tool is a vacuum nozzle which dislodges and removes simulated granular soil. An air-jet cutter has been demonstrated by others for use in field excavation.

Potentiometers and Hall Effect transducers resolve joint angles on the backhoe and arm. Their outputs are fed directly to the computer for position monitoring as well as to a controller box containing analog PID controllers. Both the backoe and arm joint angles are controlled as analog set points provided by the computer to the PID controllers.Force sensors ( strain gauges) are. deployed on 4 of the arm 's links and provide a simple hit detection mechanism. Force signals also shut down the hydraulic power supply when thresholds are exceeded.

An Intel 80286 Multibus based single board computer running the iRMX. 86 operating system, provides $\%$ real-ıme, muitı-iaskıng, interrupi ariven computing environmeni tor the excavator. An iñis graphics workstation with a high resolution color monitor is used for off-line monitoring of the process. The IRIS communicates with the Intel system to receive raw data for graphic display. It is used as a tool for assessing system performance and in hit detection for planning obstacle-free paths.

\section{SENSING}

Rex aspires to excavate pipes driven only by sensor observables. The current premap techniques are insufficient to guide secondary excavation as they lack accuracy and risk unforseen contingencies.

Because secondary excavation is basically an excercise in surface erosion, the need is for sensing simply, accurately and quickly surface state. Most modes of surface sensing were rejected (e.g. light striping, laser ranging, vision, optical proximity sensors ...) for considerations of size, inability to survive harsh environments, computing speed or non-performance in the environment of the secondary excavation. Tactile and accoustic sensors emerged as the only admissible modes and even these have their shortcomings.

Tactile sensors were considered in the form of whiskers [1], bump sensors and compliant tool holders. Whiskers were rejected for reasons of survivability and false alarms. Bump sensors either permitted too little response time between contact and crash or themselves became obstacles to desirable trajectories. An instrumented compliant nozzle is currently used as a form of compliant bump sensing. The compliance allows reasonable motion (hence time) between contact and crash, and requires no envelope space beyond that required for the nozzle alone. The instumentation on the flexible nozzle consists of 
embedded tape switch that activates when the nozzle is bent.

Acoustic sensors are utilized in secondary excavation as non-contact ranging devices. These sensors have the advantage of being rugged and compact. They provide direct $3-D$ range information about the sensed surface, bypassing any need for inferring depth from 2-D features. A disadvantage of the sonars is their inability to work with the air-knife 'on' : interference from the air-jet ovenwhelms the sonar transceiver.

The transducer of current choice (a Polaroid membrane and a Texas Instruments conditionning board) performs well on various samples of soil. Its minimum range is about $7.5^{\mathrm{m}}$ and accuracy at close range is about $0.1^{\prime \prime}$.

The data is gathered by the sonar mounied at the tip of the manipulator nex: to the tool. When the operation is in scanning mode, the motion is a back and forth sweeping in a horizontal plane over the region of interest. Each sonar reading is tagged with the 6 joint angles that exist at the time, which are used to position the adjusted reading in the model.

Sonar data is more accurate and reliable when taken closer to the surface, so the scanning motions are executed in a plane just above the highest feature of a region (as determined from the previous scan). Because the sonar transducer is axisymmetric, data is insensitive to vertical rotation. Exploiting this freedom of sensor rotation makes possible efficient scanning schemes.

\section{MODELING AND PIPE DETECTION}

The robot's world model is a data structure with access and update routines that serve as a framework for the planning and execution of operations.

The domain model is a dynamically changing surface representation: the goal of the robot being to agressively change its environment. A depth map of the region provides the necessary detail, fast access time and ease of modification.

After data gathering and building the depth map, the world modeller constructs higher level representations of the scene to represent the target objects, if any, to be cleared. Object identification exploits the fact that the target objects are limited in type : domain dependent nies are written to infer 3-D objects from $21 / 2$ - D edge lines. 
The raw sonar data is first filtered to smooth noise in the form sharp spikes or missing data. See figure 3 for a sample of the filtered data.

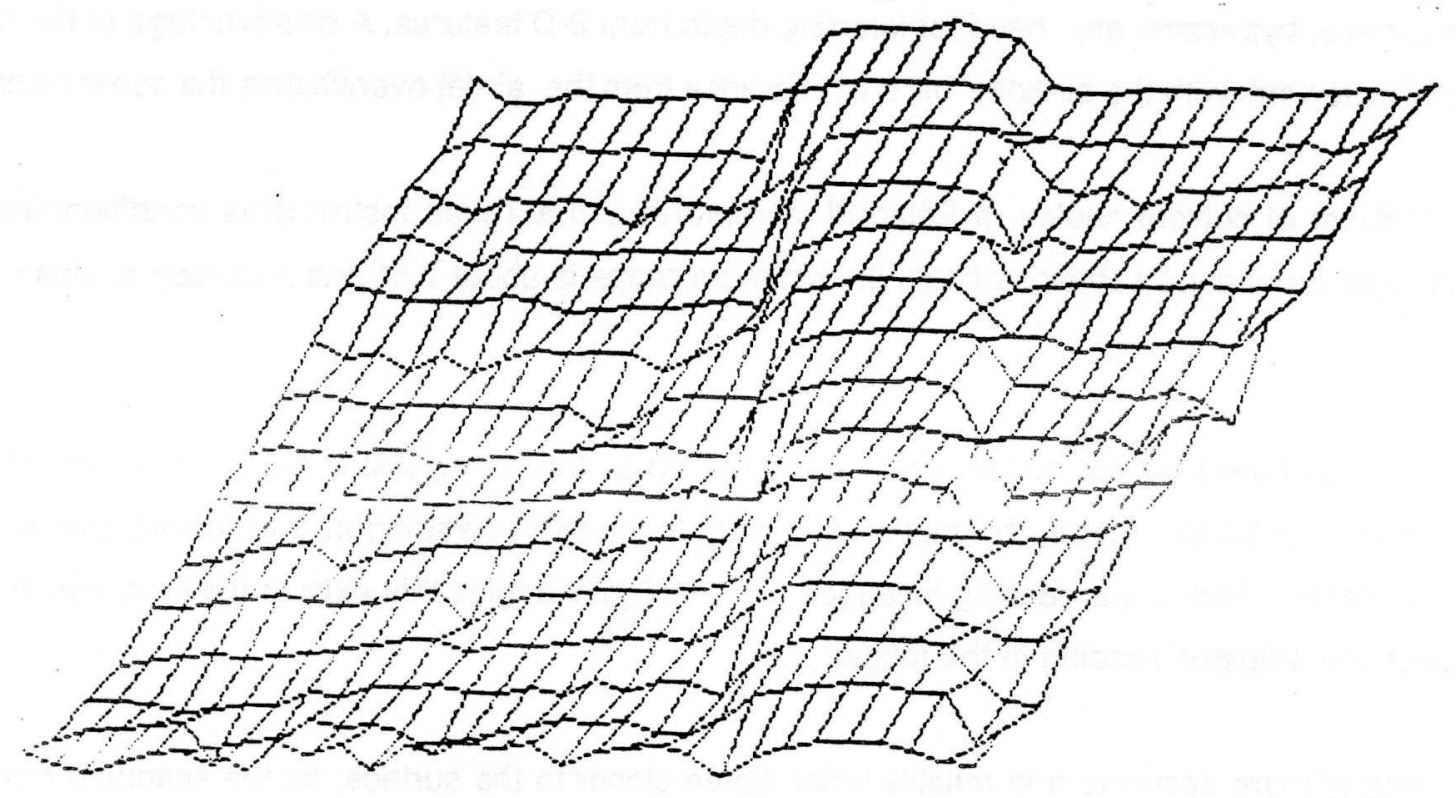

Fig 3. Sample of filtered sonar data

The next step is edge detection : A Sobel operator is applied to the data and thresholded. Typically the resulting image has thick edges. A thinning algorithm [6] is then applied to obtain single pixel edges. Line extraction from the resulting data is done by a Hough transform.

Object models are built from these edge lines. For example, two parallel lines separated by 4 to 10 inches are inferred to be a pipe. If two pipes intersect at the same depth, then depending on their continuity the target object might be an elbow, a $T$ or a cross. Object location, orientation and slope can then be directly calculated.

\section{DIGGING SCHEME}

Important features of a successful excavation strategy include execution in real time, material removal without wasted motions and ability to avoid obstacles. 
Two modes for material removal are currently available : A surface following mode is activated when no pipes are identified in the excavation scene. If pipes are present, a clearing mode is employed specifically to uncover those pipes.

The surface following strategy uses short term planning based on the topographic features of the surfa: $e$ model. This was found to be a simple and effective method of excavating while avoiding rocks and other non erodable features.

Once target objects are detected, $6^{\prime \prime}$ wide layers are excavated on both sides of the objects. This is repeated until the object is completely uncovered and cleared so that operations such as repair can take place.

\section{SIMULATION}

Graphic animation of the excavation process is provided primarily as an advisory and development tool, but the speed of the geometry engine makes possible hit detection before the execution of planned motions.

Application-level software simulates the REX environment on a Silicon Graphics Inc., IRIS model 1400 graphics workstation. This workstation consists of a $68010 \mathrm{CPU}$ and associated memory, a high resolution color monitor and an auxiliary CRT terminal. The simulation integrates data descriptions of the worksite, the manipulator, the excavation surfaces and any existing objects into a 3-D scaled, color graphics model.

Currently data representation for each of the above components can be entered into the model manually using the auxiliary terminal. In addition, manipulator position feedback from the Intel controller can be displayed in quasi-real-time (2-3 seconda latency). Sonar maps constructed from data collected during task cycles are available for post-cycle review. 


\section{DEMONSTRATIONS AND RESULTS}

First results in autonomous pipe excavation have been demonstrated in a lab simulation. The laboratory pit is filled with a granular material which covers a single straight pipe and an obstacle (a cobble stone). The pipe is detected, distinguished from the obstacle and cleared without any human intervention. Successful excavation of a $40 \times 15 \times 10$ inch volume is completed in about 15 minutes. This ability has been demonstrated with a high degree of reliability on a number of different pipe and obstacle configuration. What follows is a more specific description of the actual task.

The manipulator is positioned by the operator at a starting point above the surface of the test pit. An initial surface map is constructed from a sonar scan. This surface map is used to servo the manipulator tool tip $1 / 4$ to 1,2 inch above the soil. After a layer is removed, the map is updated by a new scan to provide new data for the succeeding pass.

As successive passes uncover the pipe, edge detection algorithms operate on the sonar data to determine pipe orientation and diameter. Once sufficient confidence in the information is achieved, the manipulator starts digging with trajectories parallel to the pipe axis. This action continues until the pipe has been sufficiently cleared.

Results to date are promising. The ability to excavate a volume, identify pipes and avoid obstacle contact has been demonstrated successfully.

\section{FUTURE WORK}

Work is in progress to further develop automated secondary excavation.

A distributed computer architecture will be implemented to achieve speed through parallelism. It will allow multi-processor, multi-tasking capabilities and distributed control of several modules.

An array of sonars will replace the single sensor used at this time. This will enable faster scans and may allow digging operations to be planned and changed on the fly.

The feature extraction and object classification will consider the gradients of the surface from layer to layer and will consider additional operators for object recognition. 
Strategy and sequencing software will be incorporated to excavate large regions containing multiple objects.

We will integrate into the world modeller and the planner the information that becomes available from premapping.

\section{ACKNOWLEDGEMENTS}

The authors are indebted to the supervision and guidance of Red Whittaker.

The Western Pennsylvania Advanced Technology Center, Southern California Gas Corporation and Dravo Corporation supported this research.

Fanners within the sponsoring institutions provided significant Dacrigrounc, insighi and formgiving.

Robotic Systems International was especially responsive in specializing manipulator hardware for this work.

Important front-end thinking was generated by student activity : David Drnevich demonstrated a compliant, instrumented tool holder. A course project by Balash, Creiman, Monley, Coffield, Peinecke and Swetz was an important precursor to the secondary excavator.

\section{REFERENCES}

[1] Agin, Gerald et al : 'Three dimensional sensing and interpretation'

Technical Report 85-1 Robotics Institute, Camegie-Mellon University.

[2] Bitz, Francois : 'REX : a remote excavator. Project Description' Intemal report - Carnegie-Mellon University, 1985.

[3] Brady, Michael et al : 'Robot Motion : planning and control' , chap 4,pp 221-304

MT press, 1982.

[4] Crowley, James: 'Dynamic world modelling for an intelligent Mobile robot using a rotating ultra-sonic ranging device'

Technical report $84-27$ Robotics Institute, Carnegie-Mellon University.

[5] Motazed, Benham : 'Inference of Ferrous Cylinders.trom Magnetic Fields'

Ph.D. Thesis, Civil Engineering department- Camegie Mellon University, Dec 84

[6] Pavlidis, Theo : 'Algorithms for graphics and image processing'

Computer Science Press, 1982

[7] Polaroid : Sonar applications notes.

[8] Sjolund, Paul and Donath Max : 'Robot task planning : Programming using interactive computer graphics' - presented at ROBOTS 7

conference. Chicago - Illinois 1983. 